\title{
Characterization of an hybrid target multi-keV x-ray source by a multi-parameter statistical analysis of Titanium K-shell emission
}

\author{
M. Primout,* D. Babonneau, L. Jacquet, and F. Gilleron \\ CEA, DAM, DIF, F-91297 Arpajon, France \\ O. Peyrusse \\ Univ Bordeaux, CELIA, UMR 5107, F-33405 Talence, France
}

\author{
K. B. Fournier, R. Marrs, M. J. May, R. F. Heeter, and R. J. Wallace \\ Lawrence National Livermore laboratory, L-41, P.O. Box 808, Livermore, California 94550, USA
}

(Dated: October 28, 2015)

\begin{abstract}
We have studied the titanium K-shell emission spectra from multi-keV x-ray source experiments with hybrid targets on the OMEGA laser facility. Using the collisional-radiative TRANSPEC code, dedicated to K-shell spectroscopy, we reproduced the main features of the detailed spectra measured with the time-resolved MSPEC spectrometer. We have developed a general method to infer the $N_{e}$, $T_{e}$ and $T_{i}$ characteristics of the target plasma from the spectral analysis (ratio of integrated Lyman$\alpha$ to Helium- $\alpha$ in-band emission and the peak amplitude of individual line ratios) of the multi-keV $\mathrm{x}$-ray emission. These thermodynamics conditions are compared to those calculated independently by the radiation-hydrodynamics transport code FCI2.
\end{abstract}

PACS numbers:

\section{INTRODUCTION}

Reliable x-ray source calculations are essential for predicting the emission level of backlighters. Efficient $\mathrm{x}$ ray sources are necessary for radiography on NIF [1][2] or LMJ [3]. Interpretation of Non Local Thermodynamic Equilibrium (NLTE) calculations of emission spectra emitted by light material from, e.g. titanium (Ti) to germanium $(\mathrm{Ge})$ have been used to infer properties of high-temperature plasmas [4][5][6][7]. Discrepancies are often observed between calculated and measured $\mathrm{x}$ ray emission from such plasmas. One reason is that the NLTE K-shell emission is calculated within a full radiation-hydrodynamics code coupled with a necessarily simplified atomic physics package [8][9]. One way to solve this disagreement is to look into more detail in the spectral data. The purpose of this paper is to analyze $\mathrm{x}$-ray source experiments with hybrid targets that took place on the OMEGA facility in February and June 2010. This analysis is a complementary approach to the broadband spectrometer (DMX[10], microDMX or DANTE) measurements that include all contributions of He-like and $\mathrm{H}$-like lines of titanium in the range 4.5 to $6.5 \mathrm{keV}$. NLTE emissivity is very sensitive to the ionization state of the plasma and particularly in this thermodynamic domain where the plasma is mainly composed of H-like and He-like ions. The Helium-like "plateau" encountered while increasing electron temperature renders the calculation very sensitive to the ionization balance. A measurement of the Lyman-alpha to Helium-alpha ratio (Ly$\alpha / \mathrm{He}-\alpha)$ is a unique opportunity to test the validity of

*Electronic address: michel.primout@cea.fr a simplified NLTE model coupled to the hydrocode by inferring thermodynamic properties of plasma from experimental spectra. The ratio of titanium Ly- $\alpha$ lines (integrated in the range $4850-5050 \mathrm{eV}$ ) to the He- $\alpha$ lines (integrated in the range $4650-4800 \mathrm{eV}$ ) is a good indication of the electronic temperature $\left(T_{e}\right)[11][12]$, provided that the electron density $\left(N_{e}\right)$ of the most emissive zone of the plasma is known. The detailed structure of the He- $\alpha$ lines, i.e. in order of increasing energies includes, for $\mathrm{Ti}(\mathrm{Z}=22)$ ions, three Li-like satellite components around $4706 \mathrm{eV}$ (named $\mathrm{S}$ ), inter combination line $\left({ }^{3} P_{1}\right)$ around $4727 \mathrm{eV}$ (named I) and resonance line $\left({ }^{1} P_{1}\right)$ about $4750 \mathrm{eV}$ (named R). Their relative amplitudes bring additional information on the thermodynamic conditions of the emissive plasma. The detailed structure of the Ly- $\alpha$ lines is composed of two He-like satellites at 4933.7 and $4943.8 \mathrm{eV}$ and the relativistic doublet ${ }^{2} P_{1 / 2}$ and ${ }^{2} P_{3 / 2}$ of $1 \mathrm{~s}-2 \mathrm{p}$ transitions at 4966 and $4977 \mathrm{eV}$, respectively. In the following, in addition to the $\mathrm{Ly}-\alpha / \mathrm{He}-\alpha$ ratio we will consider the two amplitude ratios $R / S$ and $R / I$ for comparison between measured and calculated spectra. We will map the $N_{e}-T_{e}$ plane from the calculation of the three ratios defined above as parameters in order to understand the effect of electron temperature and density on spectral structures.. The experimental conditions are briefly described in the section II B. The collisional-radiative code TRANSPEC is described in section III A. As it calculates the radiative transfer of optically thick lines, this approach will also estimate the extension of the emissive areas involved in the production of the x-ray spectra provided that we introduce a characteristic cell thickness " $e$ " as a fourth input parameter in the simulation in addition to $T_{e}, N_{e}$ and $T_{i}$. In section IV we use a statistical approach to analyze the behavior of the three output ratios with respect to the input parameters $N_{e}, T_{e}, T_{i}$ and $e$. 


\section{OMEGA EXPERIMENTS}

\section{A. The targets}

A hybrid target is comprises a titanium foil located at the end of a plastic cylinder filled with low-density aerogel $\left(2\right.$ and $5 \mathrm{mg} / \mathrm{cm}^{3}$ ). The principle can be summarized as follows: a laser incident on the front face of the aerogel confined in a plastic cylinder deposits its energy via the Inverse Bremsstrahlung process and generates a supersonic thermal wave called a "bleaching wave", which propagates through the medium and eventually reaches the titanium foil closing the cylinder. A titanium plasma is created, heated, longitudinally and radially confined both by the aerogel and the plastic cylinder. The walls of the cylinder are $100 \mu \mathrm{m}$ thick which allows transmission of the main part of multi-keV x-ray signal from the heated region to the detector. In the following, we name the targets according to their size (diameter and length of the plastic cylinder), the density of the aerogel fill and the thickness of the titanium foil. There were two lengths: L for Long $=1 \mathrm{~mm}$, S for Short $=0.5 \mathrm{~mm}$, two diameters: $\mathrm{W}$ for Wide $=2 \mathrm{~mm}, \mathrm{~N}$ for Narrow $=1 \mathrm{~mm}$, three types of filling (aerogel density $=0$, i.e. empty cylinder, 2 and 5 $\mathrm{mg} / \mathrm{cm}^{3}$ ) and two for titanium foil thicknesses (3 and 20 $\mu \mathrm{m})$. As an example: SN5-3 is a target $0.5 \mathrm{~mm}$ long and $1 \mathrm{~mm}$ in diameter, filled with $5 \mathrm{mg} / \mathrm{cm}^{3}$ density aerogel and closed by a $3 \mu \mathrm{m}$-thick titanium foil. LW0-20 is an empty target $1 \mathrm{~mm}$ long and $2 \mathrm{~mm}$ in diameter, closed by a $20 \mu m$-thick foil. The pictures in Figure 1 show a typical hybrid target as they were prepared by CEA and LLNL laboratories. The same targets were first shot on the GEKKO XII facility of the University of Osaka-Japan in November 2009 and more experimental details can be found in reference [13]. Much more detailed analysis of these targets can be found in [14].

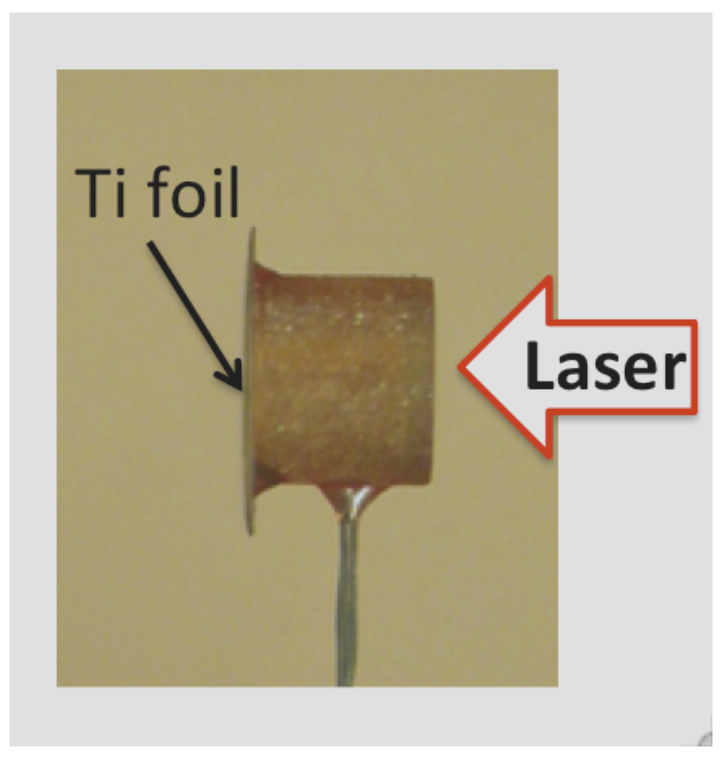

FIG. 1: Side view of an hybrid target.

\section{B. Experimental setup laser conditions and diagnostics}

In the February 2010 Omega campaign, MSPEC [15][16][17] and HENWAY [18] diagnostics were fully analyzed. The HENWAY spectrometer was affected by a significant level of noise and the data were not used for spectral analysis in addition to the fact it was a timeintegrated diagnostic. We consider only five shots whose characteristics are summarized in Table II B. The diagnostics, shown on Figures 2, are close to those of the broadband diagnostics DMX $\left(37^{\circ}\right)$ from CEA and DANTE from LLNL. For both the February and June experiments, the MSPEC was attached to a 4-strip X-ray Framing Camera with an X-ray gate width of $100+/-$ 20 ps. The MSPEC was configured with a secondgeneration elliptical PET crystal, whose spectral range spanned the $3500-6000 \mathrm{eV}$ range with spectral resolving power $E / \Delta E \approx 400$. The MSPEC was filtered with a series of filters totaling 54 microns of polypropylene $\left(C_{3} H_{6}\right.$, density $\left.0.90 \mathrm{~g} / \mathrm{cm}^{3}\right), 15$ microns of polyethylene $\left(C_{2} H_{5}\right.$, density $0.92 \mathrm{~g} / \mathrm{cm}^{3}$ ), and 1.05 microns of aluminum (density $2.7 \mathrm{~g} / \mathrm{cm}^{3}$ ). The MSPEC was fielded in spatially integrated mode (i.e. without slits) to maximize the X-ray signal/noise.

\begin{tabular}{l|l|l|l|l} 
Shot \# & Target & Ly/He-0.4 ns & Ly/He-0.7 ns & Ly/He-1.1 ns \\
\hline 56933 & LW2-3 & 0.17
\end{tabular}

\begin{tabular}{c|c|c|c|c}
\hline 56933 & LW2-3 & 0.17 & 0.19 & 0.12 \\
\hline 56934 & LW5-3 & 0.06 & 0.11 & Noise on Ly \\
\hline 56935 & SN5-20 & - & 0.06 & Noise on Ly \\
\hline 56936 & LW2-3 & 0.34 & 0.26 & 0.23 \\
\hline 56937 & SN5-20 & 0.43 & 0.38 & 0.19 \\
\hline Shot \# & Target & Ly/He-1.1 ns & \multicolumn{2}{|c}{ Ly/He-1.7 ns } \\
\hline \hline 58407 & SN2-3 & 0.12 & 0.12 (abnormal He- $\alpha)$ \\
\hline 58408 & SN2-20 & Noise on Ly- $\alpha$ & \multicolumn{2}{|c}{0.048} \\
\hline 58409 & SN5-20 & 0.11 & \multicolumn{2}{|c}{0.32} \\
\hline 58410 & LW2-3 & 0.025 weak signal & \multicolumn{2}{c}{0.27} \\
\hline 58411 & SN5-3 & 0.046 & & 0.15 \\
\hline 58412 & SN0-20 & 0.045 & & 0.074 \\
\hline
\end{tabular}

TABLE I: Experimental Ly- $\alpha / \mathrm{He}-\alpha$ ratios for all shots., Shots from February (upper) and shots for June (lower).

The six shots of the June campaign are summarized in Table II B for which HENWAY was located at an angle of $61^{\circ}$ close to that of microDMX at $63^{\circ}$. However, MSPEC was located at $101^{\circ}$ which is behind the Ti foil. The radiation from the emissive zones will cross cold $\mathrm{Ti}$, which should be taken into account when calculating the emission spectrum. We shall return to this point later in the analysis. In June, the HENWAY spectrometer was fully operational. Figure 3 shows the definition of the parameters chosen for the spectral analysis. 


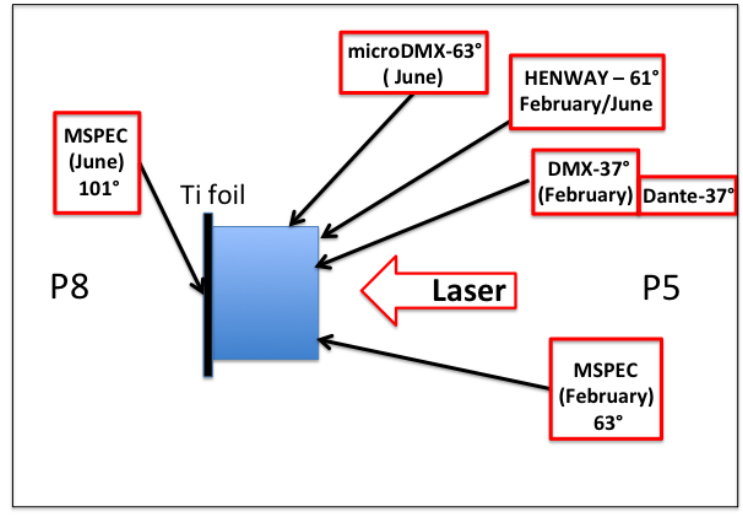

FIG. 2: Main diagnostics position in February and June on OMEGA.

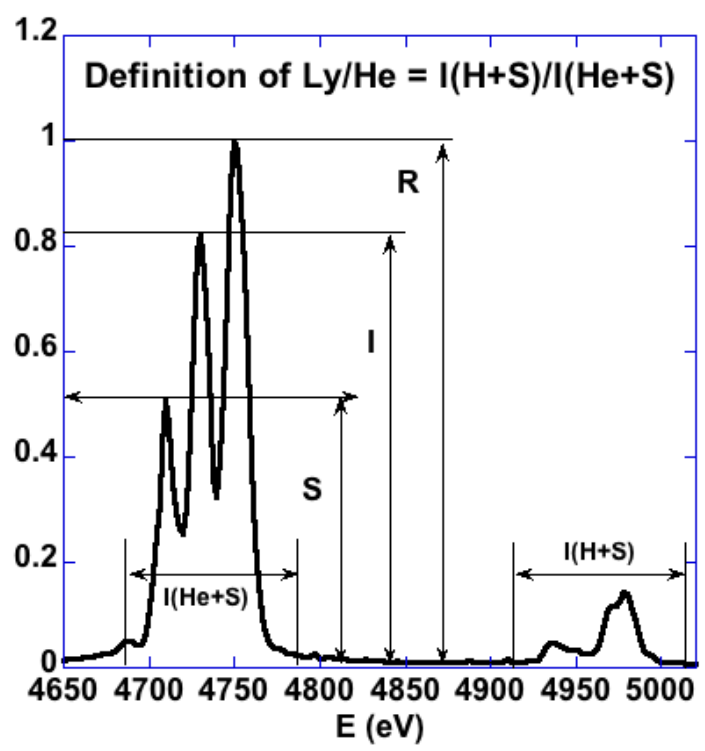

FIG. 3: Ratio definitions : Ly- $\alpha / \mathrm{He}-\alpha$ ratio of spectrum integrals between $4650-4800 \mathrm{eV}$ and $4900-5020 \mathrm{eV}, R / S$ ratio between main resonance $\mathrm{R}$ and Li-like satellite $\mathrm{S}$ and $R / I$ between main resonance and inter combination line $\mathrm{I}$.

\section{MSPEC SPECTRA ANALYSIS}

The Table II identifies the main observable transitions and the corresponding energies in the K-shell Titanium spectra.

\section{A. The TRANSPEC code}

The TRANSPEC code [19][20][21] is a tool for the spectroscopic analysis of laboratory plasmas. In its current form it is dedicated to K-shell spectroscopy. It ap-

\begin{tabular}{c|c|c}
\hline Line name & Transition levels & Energy $(\mathrm{eV})$ \\
\hline \hline Li-like satellite & $1 s^{2} 2 p-1 s\left({ }^{2} S\right) 2 p^{2}\left({ }^{3} P\right)$ & 4704.5 \\
\hline Li-like satellite & $1 s^{2} 2 p-1 s\left({ }^{2} S\right) 2 p^{2}\left({ }^{1} D\right)$ & 4710.7 \\
\hline Li-like satellite & $1 s^{2} 2 p-1 s\left({ }^{2} S\right) 2 p^{2}\left({ }^{1} D\right)$ & 4713.8 \\
\hline He- $\alpha$ Intercombination & $1 s^{2}-1 s 2 p\left({ }^{3} P_{1}\right)$ & 4727 \\
\hline He- $\alpha$ Resonance & $1 s^{2}-1 s 2 p\left({ }^{1} P_{1}\right)$ & 4750 \\
\hline He-like satellite & $1 s 2 s\left({ }^{3} S_{1}\right)-2 s 2 p\left({ }^{3} P_{\{012\}}\right)$ & 4943.8 \\
\hline He-like satellite & $1 s 2 s\left({ }^{1} S_{0}\right)-2 s 2 p\left(1 P_{1}\right)$ & 4948.7 \\
\hline Ly- $\alpha$ Doublet I & $1 s-2 p\left({ }^{2} P_{1 / 2}\right)$ & 4966 \\
\hline Ly- $\alpha$ Doublet II & $1 s-2 p\left({ }^{2} P_{3 / 2}\right)$ & 4977 \\
\hline
\end{tabular}

TABLE II: Main transitions in the K-shell Titanium spectra.

plies to a class of hot plasmas $\left(T_{e}\right.$ ranging from tens to thousands of $\mathrm{eV}$ ) and deals with ions with atomic number $\mathrm{Z}$ up to 26 . K-shell emission spectroscopy concerns mainly ions with 1, 2 or 3 bound electrons. Transitions involving an electron filling a $K$ shell vacancy $(n=1)$ in ions with more electrons are included. These transitions should nonetheless be preceeded by an excitation to an ionization level from internal processes that are unlikely in a plasma where the electrons are thermalized. When these transitions are observed, they show the presence of suprathermal electrons but this standard version of TRANSPEC applies only where the plasma electrons are thermalized. The K-shell emission has the advantage of being simple in that the strong lines are relatively few and well resolved from each other. As Z is not supposed to be very high, the profiles of these lines are more sensitive to the Stark broadening due to the plasma environment around an emitter ion. When properly addressed, the profiles therefore contain in themselves some information on the state of the plasma (electron density) and therefore TRANSPEC includes the modeling of the Stark broadening of the K-lines. In addition, the considered laboratory plasmas are open systems: the emitted photons are not necessarily reabsorbed and therefore, these plasmas are not in thermodynamics equilibrium: we must take into account all radiative and collisional processes in order to know the distribution of bound electrons. TRANSPEC contains a detailed description of the energy levels for hydrogen, helium and lithium-like ions. Other ions have only a rough description limited to the basic configuration and excited configurations corresponding to excitations of an electron up to the principal quantum number $\mathrm{n}=10$. The code works in three stages: a first step calculates the populations in different cells (i.e. plasma slab of definite thickness) and at different times, consistently with the radiation. It then generates a file containing the populations of levels belonging to different ionic species. The second step chooses a spectral range and generates the emissivities and opacities in the plasma cells defined above. The third step uses the emissivities to generate a spectral emission corresponding to a given experimental configuration. The code uses standard hypothesis of Stark line broadening, namely the quasi-static approximation for the ions and the impact 
approximation for the electrons. The microfield distribution is given by an analytic fit to numerous Monte-Carlo simulations [22] as a function of two correlation factors combining the main plasma parameters $\left(\bar{Z}, N_{e}, T_{i}, T e\right)$ :

$$
\begin{gathered}
\Gamma_{i i}=\frac{\bar{Z}^{2}}{T_{i} R_{W S}}=2.320810^{-7} N_{e}^{1 / 3} T_{i}^{-1} \bar{Z}^{5 / 3} \\
U_{i e}=\frac{R_{W S}}{\lambda_{T F}}=4.87210^{7} I_{-1 / 2}\left(\mu / T_{e}\right)^{1 / 2} N_{e}^{-1 / 3} T_{e}^{1 / 4} \bar{Z}^{1 / 3}
\end{gathered}
$$

Where $I_{-1 / 2}$ is the Fermi integral of order $-1 / 2, \mu$ is the chemical potential, $R_{W S}$ is the Wigner-Seitz mean inter ionic radius, $\lambda_{T F}$ is the Thomas-Fermi electron-ion screening length [23]. Additional broadening mechanisms include Doppler effect due to thermal motion of radiators (depending on $T_{i}$ ) and the instrumental width. The approach is fast enough to be coupled in-line with a radiative transfer calculation in an optically thick plasma, and with collisional-radiative modelling of non-LTE level populations. Lines calculated with TRANSPEC thus provide information on the interdependence of plasma properties such as $\bar{Z}, N_{e}, T_{i}$ and $T_{e}$ and the thickness $e$ of the emitting plasma. However the precision over only one particular parameter is affected by the precision of the others. Most critical is the accuracy regarding the ionic temperature which enters the ion coupling parameter $\Gamma_{i i}$ and the Doppler width, dominated by the instrumental width.

The Figure 4 shows the main features of a Ti K-shell spectrum as calculated by TRANSPEC for the conditions of an experimental MSPEC spectrum. The Li-like satellites around 5500 and $\approx 5800 \mathrm{eV}$ are not included in TRANSPEC. The observed transitions extend up to the Ly- $\gamma(1 \mathrm{~s}-4 \mathrm{p}$ transition line at $6215 \mathrm{eV})$ and above.

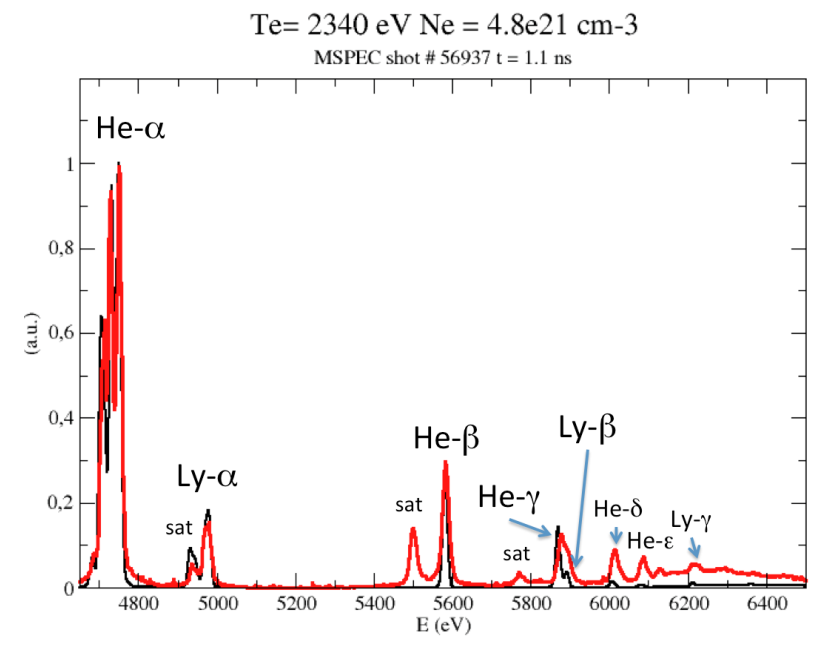

FIG. 4: Typical Titanium spectra calculated by TRANSPEC (in black) superimposed with experimental MSPEC spectrum (in red) from 4650 to $6500 \mathrm{eV}$ (February shot \#56937).

The resonance, intercombination and satellites lines of He- $\alpha$ were fitted by three gaussians. The fit was performed with only two gaussians for the Ly- $\alpha$ structure (one for the Lyman doublet and one for the He-like satellites). These fits are used to extract the values of the integrals of the components and their $\mathrm{Ly}-\alpha / \mathrm{He}-\alpha$ ratio at four times: $0.1,0.4,0.7$ and $1.1 \mathrm{~ns}$. The integrals are calculated between 4650 and $4800 \mathrm{eV}$ for the He- $\alpha$ component and between 4850 and $5050 \mathrm{eV}$ for the Ly- $\alpha$ component.

We have developed a general approach to infer electron and ionic temperature, electron density and cell thickness from experimental results. As noted above, the TRANSPEC code is able to calculate the radiative transfer in the lines through the material in the spectrometer line of sight. For this purpose we have considered the cell thickness $e$ as the fourth relevant parameter for spectral analysis.

From these spectra several thousands of $N_{e}-T_{e}-T_{i}-e$ quadruplets are chosen in a regular grid for simplicity of representation: typically $61 \times 46 x 9=25254$ for example in the respective ranges: $\left[10^{20}-10^{22} e^{-} / \mathrm{cm}^{3}\right],[1800-3000$ $\mathrm{eV}]$ and [1000-10000 eV] and a fixed value of $e=50 \mu \mathrm{m}$. We calculated the Ly- $\alpha / \mathrm{He}-\alpha$ ratios and the ratios of resonant line amplitude called $R$ to the two other lines $S$ and $I$ as detailed in the diagram in Figure 3. Our purpose is to find the quadruplet $\left(N_{e}, T_{e}, T_{i}\right.$ and $\left.e\right)$ that best reproduces the instantaneous experimental spectra. At a given time the value found are compared with results provided that by the radiation-hydrodynamics simulation. The ratios are calculated in the same way from experimental spectra and TRANSPEC's spectra. We assume that these values will be enough to characterize the emitting plasma and to give its thermodynamic properties. The calculations of the target hydrodynamics and x-ray emission have been carried out with FCI2, the CEA 2Daxisymmetric radiation-hydrodynamics computer code. For these simulations we used the RADIOM model [24] based on the ionization temperature as a simplified nonlocal-thermal-equilibrium (NLTE) model and radiation transport calculation with more than 100 photon energy groups. A ray-tracing algorithm simulates laser light propagation through matter, and the laser absorption is calculated via inverse Bremmstrahlung on free electrons. We treat the electron thermal conduction with a flux limited Spitzer-Härm formulation but we can alternatively use a nonlocal heat transport package [25]. An in-line rezoning is necessary for specific cases of empty targets (no aerogel in front of the titanium foil) especially for the longest pulses. Power emitted in the 4-6 $\mathrm{keV}$ titanium $\mathrm{K}$-shell band in the angular directions of diagnostics (37 $7^{\circ}$ for DMX at OMEGA in February, $63^{\circ}$ for $\mu \mathrm{DMX}$ at OMEGA in June) and spectral power for spectroscopy diagnostics $\left(63^{\circ}\right.$ for MSPEC in February, $101^{\circ}$ in June, $61^{\circ}$ for HENWAY both campaigns), have been calculated with FCI2 and the diagnostic post processor DIXIM [26]. The HENWAY data exhibit a lot of noise in February so there are analysed further.

Postprocessing the FCI2 code with DIXIM enables us to find the spatial origin for photons reaching the MSPEC spectrometer in the range $4-6 \mathrm{keV}$. For the February 
shots, the zone of maximum contribution to the spectra is located in the outer part of the expanding titanium foil, i.e. the emission zone responsible for the main MSPEC spectrum features can be restricted to a very small number of cells with specific $N_{e}, T_{e}, T_{i}$ and $e$ values (Figures 5). We assume that the spectrum recorded at each time by MSPEC mainly comes from this most emissive zone determined by FCI2. Clearly, this assumption is less acceptable for June shots, where, as mentioned above, MSPEC is looking at the rear-side of the Titanium foil.

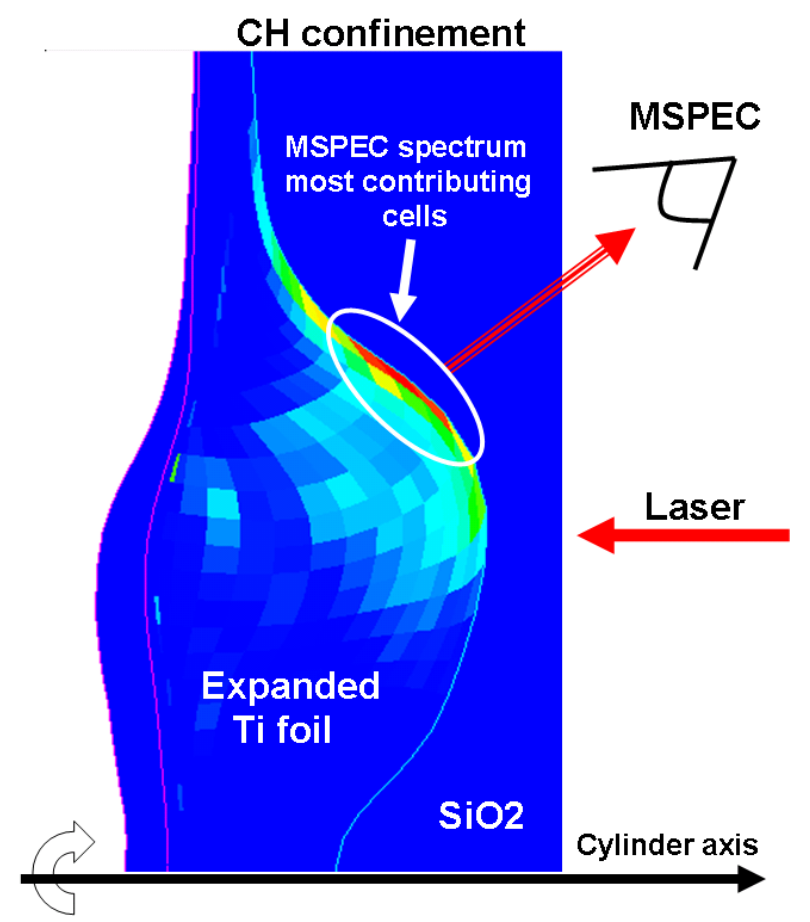

FIG. 5: Color plot of photon origin at $0.7 \mathrm{~ns}$ : most heavily contributing zone to the MSPEC spectrum in the range 4-6 $\mathrm{keV}$.

\section{B. Isovalues of $\mathbf{L y}-\alpha / \mathbf{H e}-\alpha, R / S$ and $R / I$ ratios}

For each value of the ratio $\mathrm{Ly}-\alpha / \mathrm{He}-\alpha$, it is possible to determine a path in the $N_{e} / T_{e}$ plane corresponding of that isovalue. The Figure 6 shows isovalues of Ly$\alpha /$ He- $\alpha$ from 0.06 to 0.12 and $R / S$ from 1 . to 1.8 in the $N_{e}-T_{e}$ plane. These contours are drawn for fixed values of $T_{i}$ and $e$. An intersection is always found between the Ly- $\alpha / \mathrm{He}-\alpha$ and $R / S$ contours. In the following we show how the ionic temperature modifies the line ratios.

We start with the most restrictive condition, i.e. the Ly- $\alpha /$ He- $\alpha$ ratio which gives us a parametric relationship between $N_{e}$ and $T_{e}$. In a second step, we look in more detail at the relative amplitudes of the three He- $\alpha$ main components (S, R and I). The Li-like satellite feature comprises three lines at 4704.5, 4710.7 and 4713.8

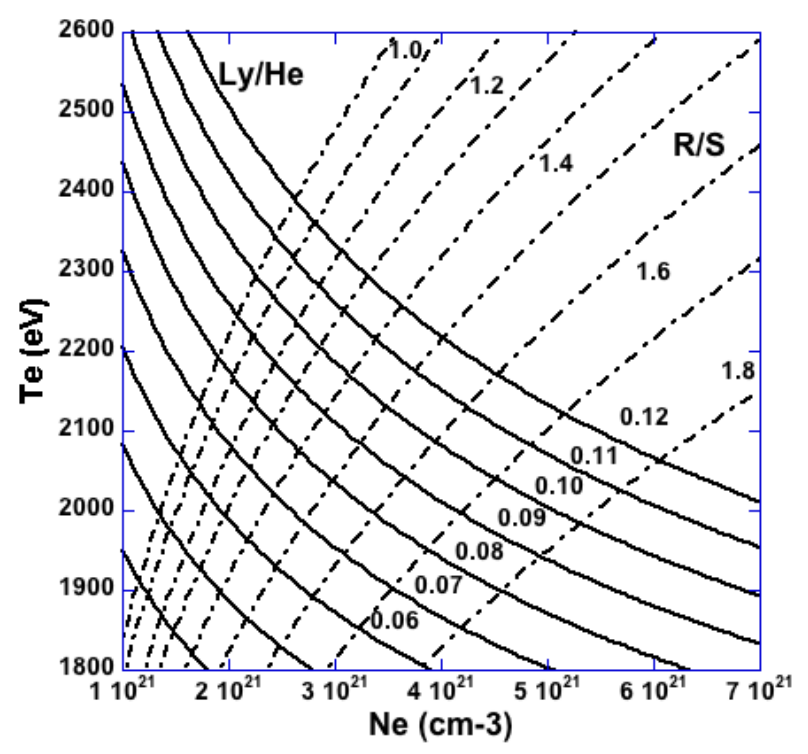

FIG. 6: Isovalues of the Ly- $\alpha / \mathrm{He}-\alpha$ and $R / S$ ratios in $N_{e}-T_{e}$ plane, for given values of $T_{i}$ and $e\left(T_{i}=T_{e}\right.$ and $\left.e=50 \mu \mathrm{m}\right)$. The lines have been fitted with power laws in Ly- $\alpha / \mathrm{He}-\alpha$ (solid lines) and $R / S$ (dot-dashed lines).

$\mathrm{eV}$, which are responsible for the shoulder observed on the He- $\alpha$ line shape. Figure 7 shows TRANSPEC calculations with and without experimental resolution. The same pattern is observed for the He-like satellite of the Ly- $\alpha$ structure in Figure 8 for 4933.7, 4943.8 and 4948.7 $\mathrm{eV}$ respectively. This asymmetric shape is clearly observable in experimental spectra.

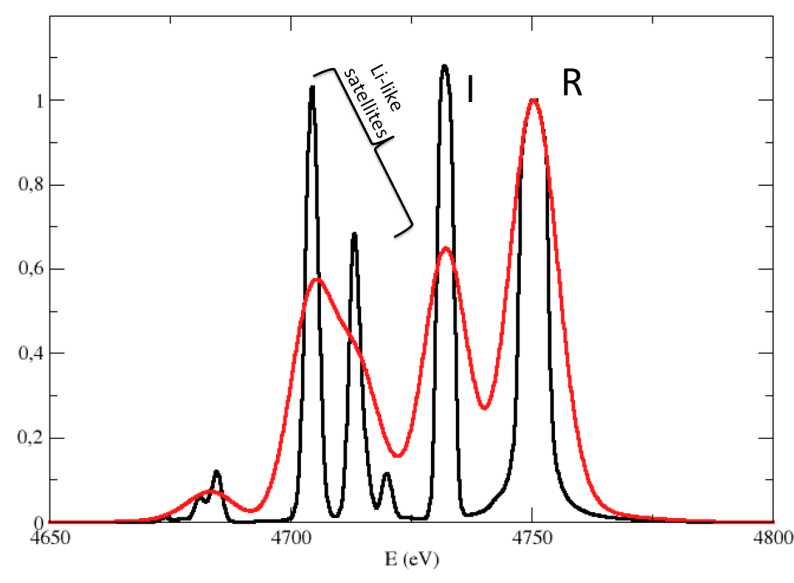

FIG. 7: Effect of experimental resolution, as calculated by TRANSPEC, on the Li-like satellites of He- $\alpha$ structures (red: resolution of $7 \mathrm{eV}$, black: no experimental resolution).

We calculated the $R / S$ and $R / I$ ratios from experimental spectra for all OMEGA shots (for different times: 0.4, 0.7 and $1.1 \mathrm{~ns}$ in February and 1.1 and $1.7 \mathrm{~ns}$ in June). The results are summarized in Table III and Table IV. The ratios deduced from the experimental spectra have the following ranges: $0.045 \leq \mathrm{Ly}-\alpha / \mathrm{He}-\alpha \leq 0.38$, 


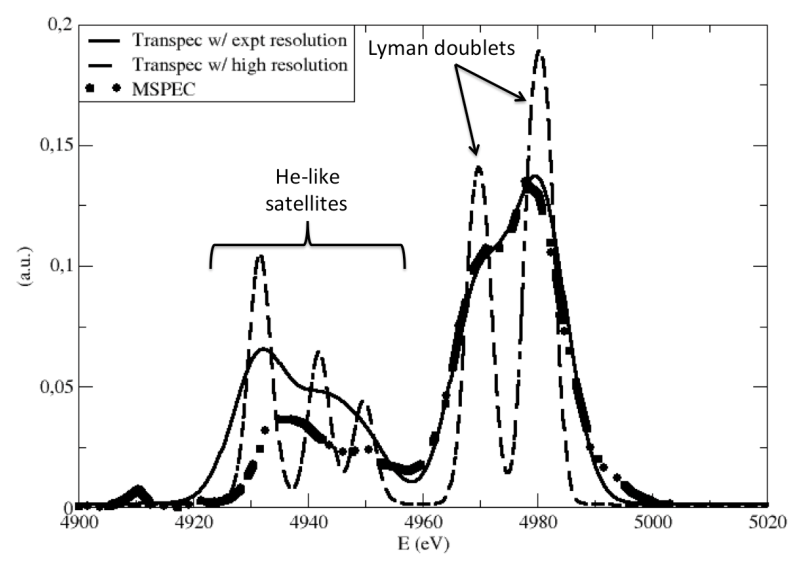

FIG. 8: Effect of experimental resolution, as calculated by TRANSPEC, on the He-like satellites of Ly- $\alpha$ structures (dash black: resolution of $7 \mathrm{eV}$, black: no experimental resolution). Experimental spectrum (dot-dash-bold) is superimposed.

\begin{tabular}{c|c|c|c|c|c|c|c}
\hline $\begin{array}{c}\text { Shot } \\
\#\end{array}$ & $\begin{array}{c}\text { Target } \\
\text { type }\end{array}$ & $0.4 \mathrm{~ns}$ & $\begin{array}{c}R / S \\
0.7 \mathrm{~ns}\end{array}$ & $1.1 \mathrm{~ns}$ & $0.4 \mathrm{~ns}$ & $\begin{array}{c}R / I \\
0.7 \mathrm{~ns}\end{array}$ & $1.1 \mathrm{~ns}$ \\
\hline \hline 56933 & LW2-3 & 1.73 & 1.884 & 1.623 & 1.109 & 1.204 & - \\
\hline 56934 & LW5-3 & 1.68 & 1.697 & - & 1.185 & 1.222 & - \\
\hline 56935 & LW5-20 & 1.26 & 1.483 & - & 1.089 & 1.230 & - \\
\hline 56936 & SN5-20 & 1.62 & 1.786 & 1.428 & 1.081 & 1.139 & 1.047 \\
\hline 56937 & SN2-3 & 1.709 & 1.844 & 1.574 & 1.192 & 1.153 & 1.063 \\
\hline & \multicolumn{10}{|c}{}
\end{tabular}

TABLE III: He-like R to Li-like satellite $\mathrm{S}$ or intercombination I line ratios from for shots in the 2010 OMEGA February campaign. Ratios with '-' are due to anomalous line spectral features compromising either $R / S$ or $R / I$ ratio.

\begin{tabular}{c|c|c|c|c|c}
\hline $\begin{array}{c}\text { Shot } \\
\#\end{array}$ & $\begin{array}{c}\text { Target } \\
\text { type }\end{array}$ & $\begin{array}{c}R / S \\
1.1 \mathrm{nn}\end{array}$ & $\begin{array}{c}R / S \\
1.7 \mathrm{~ns}\end{array}$ & $\begin{array}{c}R / 1 \\
1.1 \mathrm{~ns}\end{array}$ & $\begin{array}{c}R / I \\
1.7 \mathrm{~ns}\end{array}$ \\
\hline \hline 58407 & SN2-3 & 3.74 & 2.03 & 1.363 & 1.370 \\
\hline 58408 & SN2-20 & 3.465 & 4.287 & 1.313 & 1.709 \\
\hline 58409 & SN5-20 & 2.036 & 2.493 & 1.289 & 1.305 \\
\hline 58410 & LW2-3 & 1.801 & 1.362 & 1.116 & 1.096 \\
\hline 58411 & SN5-3 & 2.587 & 1.996 & 1.114 & 1.222 \\
\hline 58412 & SN0-20 & 2.508 & 3.044 & 1.468 & 1.553 \\
\hline
\end{tabular}

TABLE IV: Characteristics ratios from shots in the 2010 OMEGA June campaign, similar to table III.

\section{The effect of ionic temperature on the $R / I$ ratio}

The intersection of Ly- $\alpha / \mathrm{He}-\alpha$ and $R / S$ isovalues give an initial estimate of the electron conditions. The ion temperature will be necessary to make the value of the ratio $R / I$ intersect the first point. In all cases this solution has been found (Figure 9). The ionic temperature has a small influence on the first two ratios Ly- $\alpha / \mathrm{He}-\alpha$ and $R / S$ but a much stronger one on the $R / I$ ratio. The evolution of the ratio $R / I$ with $T_{e}$ and $N_{e}$ shows that non negligeable $T_{i}$ values are necessary to reproduce the experimental values $(R / I \leq 1.5)$. Many shots exhibit a value around 1.2. This is a signature of ionic environment effect and the ion temperature whose role through the correlation parameter $\Gamma_{i i}$ has already been invoked in section III A. This overall effect of $T_{i}$ can be shown by plotting the evolution of the amplitudes $\mathrm{R}$ and I and of their ratio with ionic temperature (Figure 10), for a given density and cell thickness. The statistical analysis of the section IV will show Pareto fronts of the $R / I$ versus $T_{i}$ and $R / S$ versus $N_{e}$ ratios, confirming its influence on the global fit of experimental spectra.

In most cases, the relative amplitude of $\mathrm{He}-\alpha$ resonance line is always greater than the intercombination (I) and satellite amplitude $(\mathrm{S})$. The ratio $R / I$ gives an indication of the maximum range of $T_{i}$. Note the presence of the aerogel in front of th titanium foil affects the ion temperature: when expanding into the hot aerogel, the titanium plasma is confined by both the plastic cylinder and the aerogel plasma and this effect is often more pronounced $e$ values. 


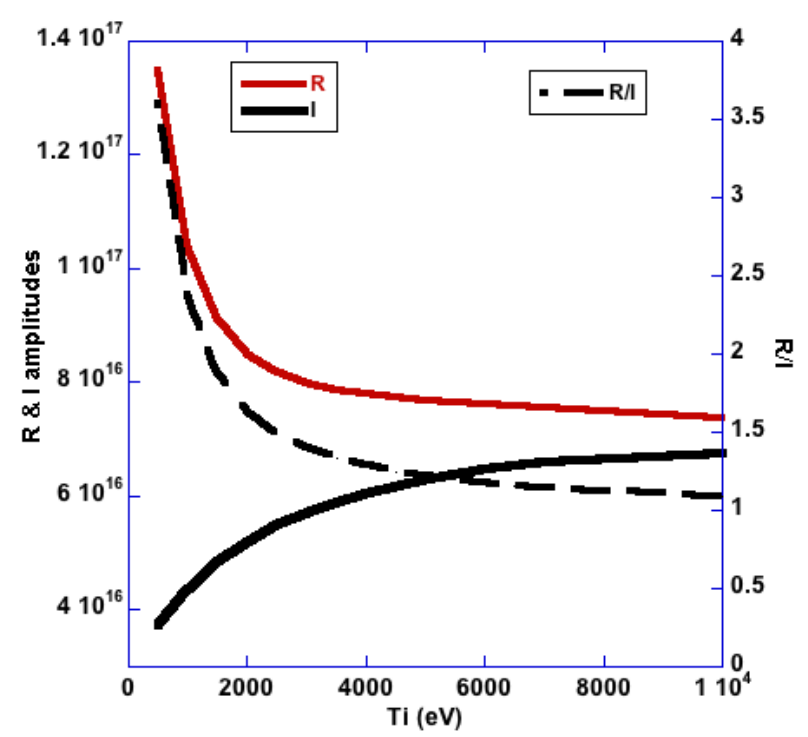

FIG. 10: Evolution of the amplitudes of resonance R (red line) and intercombination lines I (black line) of He- $\alpha$ structures and their ratios R/I (dash black line) with ionic temperature as calculated by TRANSPEC.

when the aerogel density increases. This hydrodynamic heating increases the ionic temperature or outer zones of the titanium foil. We assumed that these outer regions of the titanium plasma are responsible for the majority of the measured x-ray energy on MSPEC spectrometer.

\section{STATISTICAL APPROACH}

We show in this paragraph how a probabilistic approach can reveal significant information from the spectral data analysis. In particular it gives information about correlations between parameters that we can deduce from a large number of simulations. Table $\mathrm{V}$ displays the $8 \times 8$ correlation matrix for the four input parameters $\left(N_{e}, T_{e}, T_{i}\right.$ and e) and the four output ones ( $\mathrm{Z}^{*}$, Ly$\alpha / \mathrm{He}-\alpha, R / S$ and $R / I$ ), as calculated by TRANSPEC). As expected, there is a strong correlation (0.886) between Ly- $\alpha / \mathrm{He}-\alpha$ and the electronic temperature $T_{e}$ and an even stronger one with the ionization $Z^{*}(0.926)$. The correlation with the electronic density is weaker $(0.318)$. On the contrary the parameters $R / S$ and $R / I$ are weekly correlated ( 0.381 and 0.183 respectively) to Ly- $\alpha / \mathrm{He}-\alpha$ ratio whereas they are strongly correlated to each other (0.809). As already mentioned the $R / I$ ratio depends strongly on $T_{i}$ with an anticorrelation coefficient of 0.442 whereas the other two ratios weakly depend on the ionic temperature $T_{i}$ with only 0.065 correlation factor for $R / S$ and 0.01 for Ly- $\alpha /$ He- $\alpha$. This correlation matrix $V_{i j}(3 \times 3$ sub matrix elements are in bold characters in Table V) allows us to calculate a generalized $\chi^{2}$, taking into account the correlation between parameters, of the form:

$$
\chi^{2}=\Sigma_{i, j=1}^{3}\left(\alpha_{i}^{e x p}-\alpha_{i}^{c a l c}\right) V_{i j}\left(\alpha_{j}^{e x p}-\alpha_{j}^{c a l c}\right)
$$

where $\alpha_{1}=$ Ly- $\alpha /$ He- $\alpha, \alpha_{2}=R / S$ and $\alpha_{3}=R / I$. When the three parameters are not correlated, $V_{i j}=\delta_{i j}$, the expression for $\chi^{2}$ simplifies to $\Sigma_{i=1}^{3}\left(\alpha_{i}^{e x p}-\alpha_{i}^{\text {calc }}\right)^{2}$. In a first step we deal with 10000 Monte-Carlo draws in definite ranges for input parameters $N_{e}, T_{e}, T_{i}$ and thickness $e$. We then study the variations of the ratios Ly- $\alpha / \mathrm{He}-$ $\alpha, R / S$ and $R / I$ with these data. In a second step, we plot all the two by two combinations of the four input parameters : $T_{e}$ and $N_{e}, T_{e}$ and $e, T_{e}$ and $T_{i}, T_{i}$ and $N_{e}, T_{i}$ and $e$ and $N_{e}$ and $e$ in a restricted domain around the minimum value of $\chi^{2}$, e.g. $\chi^{2} / \chi_{\min }^{2} \leq 4$. This shows for example that the relevant parameter for spectrum description is not the electronic density $N_{e}$ nor the thickness of plasma cell $e$ but the product of both parameters, i.e., the areal density. We show these correlations on Te$\mathrm{Ne}$ (Figure 11-upper-left), e-Ne (Figure 11-upper-right) and on Te-Ne*e (Figure 11-bottom-left). The near-tominimum $\chi^{2}$ constraint acts only on the electronic temperature but not on the electron density. The relation between $e$ and $N_{e}$ can be fitted by a power law of the type $N_{e} \approx e^{-1.13}$ (Figure 11-bottom-right). The background points are the uniform Monte Carlo sample and the superimposed red points correspond to eight values near the minimum $\chi^{2}$. It is interesting to plot the spectra calculated by TRANSPEC in the eight conditions picked up from the Figure 11-lower-left. We have done so in 11, the $T_{e}-N_{e}$ pairs are tabulated in the figure. These values correspond to values of parameters giving the eight lowest values of $\chi^{2}$ around the mimimum and used in the fit of Figure 11-bottom-right, so it explains why the spectra are so similar, differing slightly by intercombination and satellite line relative amplitudes. It is intrinsically impossible to determine $N_{e}$ and $e$ independently, but their product is seen to be relevant from the spectral data characterization.

\begin{tabular}{l|c|c|c|c|c|c|c|c}
\hline & $\mathrm{Ne}$ & $\mathrm{Te}$ & $\mathrm{Ti}$ & $\mathrm{e}$ & $\mathrm{Z}^{*}$ & $L / H$ & $R / S$ & $R / I$ \\
\hline \hline$N_{e}$ & 1. & 0.004 & -0.001 & 0.004 & 0.109 & 0.318 & -0.389 & -0.395 \\
\hline$T_{e}$ & 0.004 & 1. & -0.004 & 0.009 & 0.988 & 0.886 & 0.715 & 0.486 \\
\hline$T_{i}$ & -0.001 & -0.004 & 1. & -0.001 & -0.010 & -0.001 & -0.065 & -0.442 \\
\hline $\mathrm{e}$ & 0.004 & 0.009 & -0.001 & 1. & 0.018 & 0.169 & -0.282 & -0.293 \\
\hline $\mathrm{Z}^{*}$ & 0.109 & 0.988 & -0.010 & 0.018 & 1. & 0.926 & 0.669 & 0.438 \\
\hline$L / H$ & 0.318 & 0.886 & -0.001 & 0.169 & 0.926 & $\mathbf{1 .}$ & $\mathbf{0 . 3 8 1}$ & $\mathbf{0 . 1 8 3}$ \\
\hline$R / S$ & -0.389 & 0.715 & -0.065 & -0.282 & 0.669 & $\mathbf{0 . 3 8 1}$ & $\mathbf{1 .}$ & $\mathbf{0 . 8 0 9}$ \\
\hline$R / I$ & -0.395 & 0.486 & -0.442 & -0.293 & 0.438 & $\mathbf{0 . 1 8 3}$ & $\mathbf{0 . 8 0 9}$ & $\mathbf{1 .}$ \\
\hline
\end{tabular}

TABLE V: Correlation coefficients between $N_{e}, T_{e}, T_{i}$, e and the 3 ratios $\alpha_{i}$ (from a set of 10000 Monte-Carlo draws).

\section{A. Evaluation of the method and error analysis}

To evaluate the uncertainty inherent to our method we calculated thousands of TRANSPEC spectra with known random input values of $T_{e}, N_{e}, T_{i}$ and $e$. The reconstruction procedure, based on the calculation of three ratios Ly- $\alpha /$ He- $\alpha, R / S$ and $R / I$ is applied to each spec- 

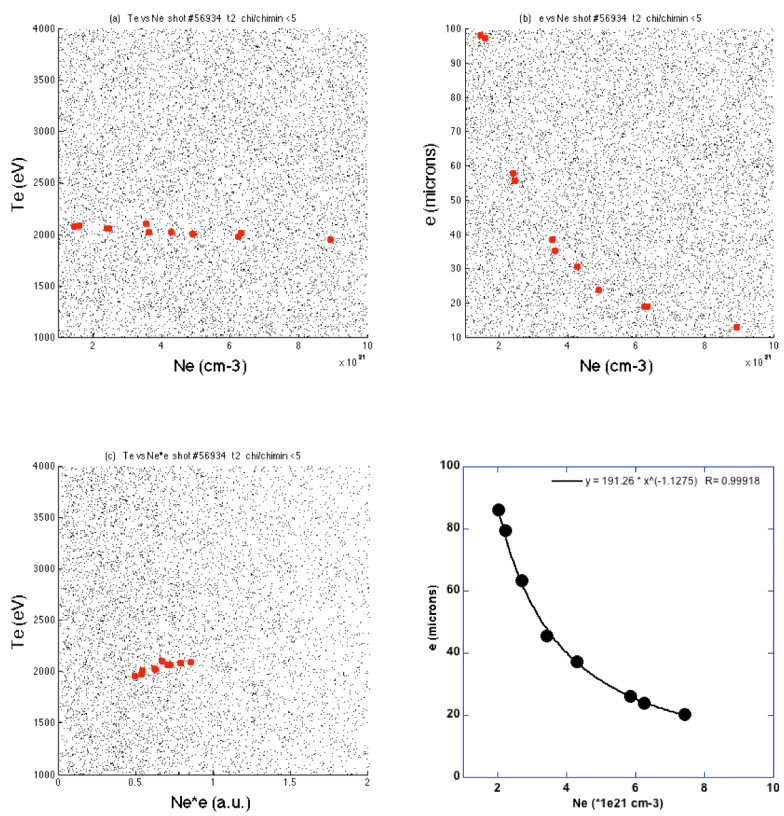

FIG. 11: $T_{e}$ vs $N_{e}$ (upper-left), $e$ vs $N_{e}$ (upper-right), $T_{e}$ vs $N_{e} * e$ (bottom-left): background black points are uniform Monte Carlo sample and eight red points are only for which $\chi^{2}$ around $\chi_{\min }^{2}$. Power law fit (bottom-right picture) involving the red points gives the relation $e \propto N_{e}^{-1.13}$.

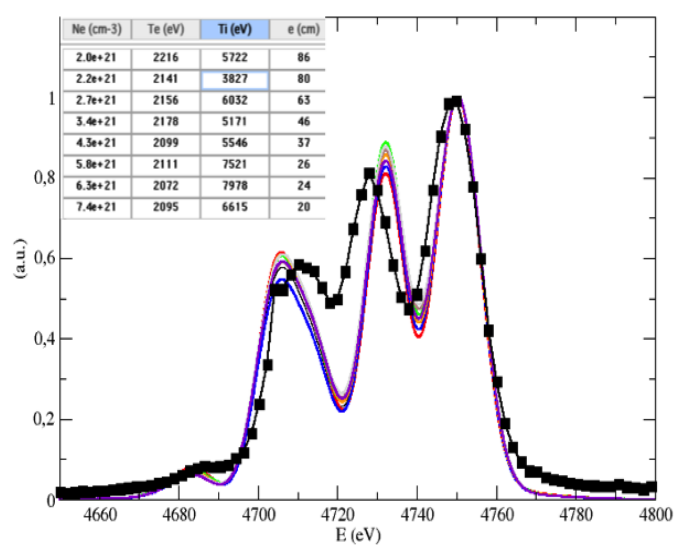

FIG. 12: Comparisons of spectra near $\chi_{\min }^{2}$ calculated by TRANSPEC and superimposed with an experimental MSPEC spectrum: He- $\alpha$ lines. The eight quadruplets considered for $N_{e}, T_{e}, T_{i}$ and $e$ are in the left-upper part of the pictures.

trum. Then, we minimize the $\chi^{2}$ within a precomputed sample based on 20000 independent Monte-Carlo samples within the same input parameter ranges. Figure 13 shows the relative errors between the known input values of $T_{e}^{i n}, T_{i}^{i n},\left(e * N_{e}\right)^{i n}$ and the calculated ones, e.g., $100 *\left(T_{e}^{c a l c}-T_{e}^{i n}\right) / T_{e}^{i n}$ for $T_{e}$. The error distributions for

\begin{tabular}{c|c|c|c|c|c}
\hline $\begin{array}{c}\text { Shot } \\
\#\end{array}$ & $\begin{array}{c}\text { Time } \\
(\mathrm{ns})\end{array}$ & $\begin{array}{c}N_{e} \\
\left(10^{21} \mathrm{e}^{-} / \mathrm{cm}^{3}\right)\end{array}$ & $\begin{array}{c}T_{e} \\
(\mathrm{eV})\end{array}$ & $\begin{array}{c}T_{i} \\
(\mathrm{eV})\end{array}$ & $\begin{array}{c}e \\
(\mu \mathrm{m})\end{array}$ \\
\hline 56933 & 0.4 & 2.7 & 2328 & 5563 & 77 \\
56933 & 0.7 & 3.7 & 2384 & 4762 & 55 \\
56933 & 1.1 & 3.2 & 2357 & 5942 & 75 \\
\hline 56934 & 0.4 & 2.2 & 1984 & 5616 & 58 \\
56934 & 0.7 & 4.6 & 2058 & 5211 & 31 \\
56934 & 1.1 & - & - & - & - \\
\hline 56935 & 0.4 & - & - & - & - \\
56935 & 0.7 & 1.8 & 1868 & 3869 & 71 \\
56935 & 1.1 & - & - & - & - \\
\hline 56936 & 0.4 & 6.1 & 2593 & 5648 & 55 \\
56936 & 0.7 & 4.4 & 2469 & 5592 & 56 \\
56936 & 1.1 & 3.7 & 2261 & 5673 & 67 \\
\hline 56937 & 0.4 & 5.7 & 2544 & 4644 & 53 \\
56937 & 0.7 & 6.1 & 2504 & 5750 & 41 \\
56937 & 1.1 & 3.2 & 2357 & 5942 & 75 \\
\hline
\end{tabular}

TABLE VI: Results for TRANSPEC calculations on February shots.

$T_{e}, T_{i}$ and the product $e * N_{e}$ are fitted by gaussians with standard deviations equal to $3.3 \%, 24.7 \%$ and $13.4 \%$, respectively. The mean values are equal to $0.01 \%,-0.003$ $\%$ and $0.03 \%$, respectively. This is the minimum error one can associate to each value. One has to add the construction error due to imperfect experimental data. In addition, the method proved to be unbiased as all the mean values are very close to zero. As already mentioned, the electronic temperature is calculated with a much better accuracy than other parameters. The electron density only would have been calculated with a poor accuracy of $62 \%$, that is why the result is given for the product $e * N_{e}$ for which the error, as already shown above, is $13.4 \%$.

In addition to the estimation of intrinsic reconstruction errors for each quantity of interest, this method enables us to give an evaluation of the accuracy of the $\chi^{2}$ minimization procedures. As an illustration we plot this only for electronic temperature and the Table VI gives the standard deviation on $T_{e}$ associated to five extended $\chi^{2}$ definitions. The definition \#3 is the most accurate one, with near-zero mean value although the other $\chi^{2}$ definition, except for method \#4, is not so bad.

On Figure 16 the shapes of $T_{e}, T_{i}$ and $N_{e} * e$ evolution curves from $\chi^{2}$ minimization procedure for shot \#56934 correspond to the uncertainty on the $T_{e}, T_{i}$ and $N_{e} * e$. The $T_{i}$ curve is less symmetric than the $T_{e}$ one and the precision on $T_{e}$ is twice as better as for ionic temperature.

The Monte-Carlo calculations span a larger range of parameters, e.g. $T_{e}=1000-4000 \mathrm{eV}, T_{i}=1000-10000$ $\mathrm{eV}, N_{e}=10^{21}-10^{22} e^{-} / \mathrm{cm}^{3}$ and cell thickness $e=10-$ $100 \mu \mathrm{m}$. The Figure 17-(a) shows that the higher the Ly- $\alpha / \mathrm{He}-\alpha$ ratio the higher the uncertainty on electronic temperature. Figures 17-(d)(h)(l) show a rather slow dependence of the three ratios on the cell thickness. Picture 17-(g) shows the correlation as mentioned above between the two amplitudes ratios $R / I$ and $R / S$. Picture 17 -(f) shows the effect of the ionic temperature on the $R / I$ ra- 


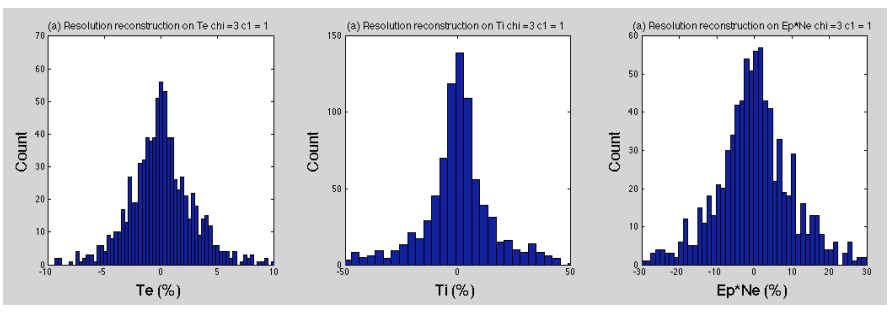

FIG. 13: Resolution on $T_{e}, T_{i}$ and $N_{e} * E_{p}$ (from left to right).

\begin{tabular}{c|c|c|c}
\hline$\#$ & $\chi^{2}$ definition & $\sigma(\%)$ & $<m>(\%)$ \\
\hline \hline 1 & $\sum_{i, j=1}^{3}\left(\alpha_{i}^{e x p}-\alpha_{i}^{\text {calc }}\right) V_{i j}\left(\alpha_{j}^{\text {exp }}-\alpha_{j}^{\text {calc }}\right)$ & 3.8 & -0.024 \\
\hline 2 & $\sum_{i=1}^{3}\left(\alpha_{i}^{e x p}-\alpha_{i}^{\text {ealc }}\right)^{2}$ & 3.9 & -0.003 \\
\hline 3 & $\sum_{i=1}^{3}\left(\alpha_{i}^{e x p}-\alpha_{i}^{\text {calc }}\right)^{2} / \alpha_{i}^{c a l c}$ & 3.3 & -0.07 \\
\hline 4 & $\sum_{i=1}^{3}\left(\alpha_{i}^{\text {exp }}-\alpha_{i}^{\text {calc }}\right)^{2} /\left(\alpha_{i}^{\text {calc }}\right)^{2}$ & 8.6 & -0.8 \\
\hline 5 & $\sum_{i=1}^{3} \alpha_{i}^{\text {calc }}\left(\alpha_{i}^{\text {exp }}-\alpha_{i}^{\text {calc }}\right)^{2}$ & 3.1 & -0.16 \\
\hline
\end{tabular}

TABLE VII: Resolution on $T_{e}$ for various $\chi^{2}$ methods: $\alpha_{i}$ stand for Ly- $\alpha / \mathrm{He}-\alpha, R / S$ and $R / I$.

tio and figure 17-(e) shows that values lower than 1 can only occur for electron temperature under $3000 \mathrm{eV}$. Let us look into detail how to interpret the Pareto fronts from picture 17-(i) for $R / S-T_{e}$ and picture 17-(f) for $R / I-T_{i}$ : on Figure 14 value of $R / I$ ratio as low as $\approx 1.2$ will never be obtained with values of $T_{i}$ less than $3000 \mathrm{eV}$ whatever the other parameters. On Figure 15, on the contrary values of $R / S$ as high as $\approx 1.5$ will never be reached with values of $T_{e}$ greater that $3000 \mathrm{eV}$. In both cases, the red dot line is a fourth-degree-polynomial fit of the Pareto front e.g. for example $R / I=a_{1} T_{i}^{4}+a_{2} T_{i}^{3}+a_{3} T_{i}^{2}+a_{4} T_{i}+$ $a_{5}$ with $a_{1}=5.302610^{-15}, a_{2}=-9.195510^{-11}, a_{3}=$ $6.045910^{-7}, a_{4}=-0.0019$ and $a_{5}=3.4481$.

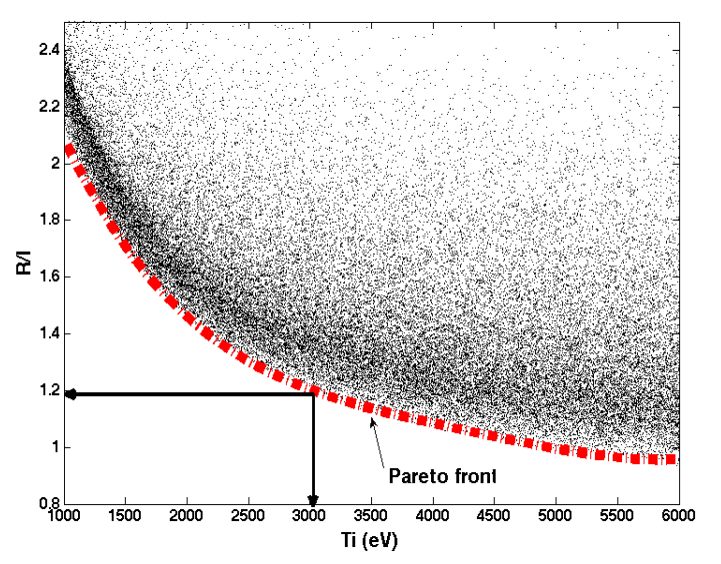

FIG. 14: Pareto front for $R / I$ ratio versus ionic temperature $T_{i}$ (red dashed curve).

For given values of the electronic temperature we can derive the evolution of the $\chi^{2}$ with the electron density and vice-versa. Pictures on Figure 19 illustrate the best

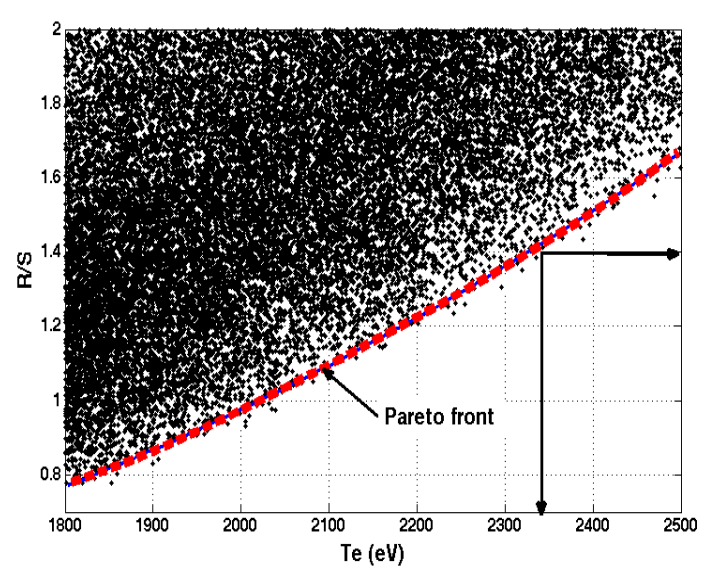

FIG. 15: Pareto front for $R / S$ ratio versus ionic temperature $T_{e}$ (red dashed curve).

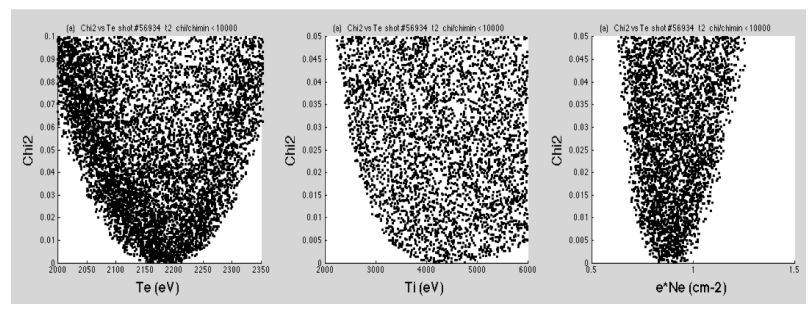

FIG. 16: $\chi^{2}$ versus $T_{e}, T_{i}$ and $N_{e} * e$ for shot \#56934.

fit TRANSPEC spectrum with the MSPEC spectrum of the shots \#56934 and \#56937 at 0.7 ns. The Ly- $\alpha$ structure is very well reproduced.

As an illustration we show the results from radiativehydrodynamics simulation for one February shot \#56934. We calculated the emissive zones responsible for x-ray in the range 4-6 keV in the direction of MSPEC diagnostic. The radiative-hydrodynamics FCI2 was post-processed by DIXIM code and we already showed a colormap for photon origin in $4-6 \mathrm{keV}$ on Figure 5 of section III. For this shot we observed a predominant zone at the outer layer of titanium foil. Figure 18 shows the colormaps of $T_{i}$ (upper left), $T_{e}$ (upper right), $N_{e}$ (lower left) and origin of photons in 4-6 keV (lower right). Note that these most emitting zones do not correspond to the highest electron temperature zone (which is much closer to the symmetry axis). The simulation shows that the most emitting cells corresponds also to a maximum value for ratio $T_{i} / T_{e}$, of the order of 1.5. The agreement is not so bad and the ionic temperature necessary to reproduce relative amplitude $R / I$ is compatible with FCI2 and TRANSPEC results. From Figure 16, the uncertainty on $N_{e}$ is three times higher that on $T_{e}$. If we consider the values density and temperature in the few cells that contributed within, e.g., $50 \%$ of the maximum x-ray emission in the range 4-6 $\mathrm{keV}$, the hypothesis of a dominant emitting zone at the outer layer of the Titanium foil seems to be valid and the values derived from TRANSPEC is in rather good 
(a) LylHe vs Te

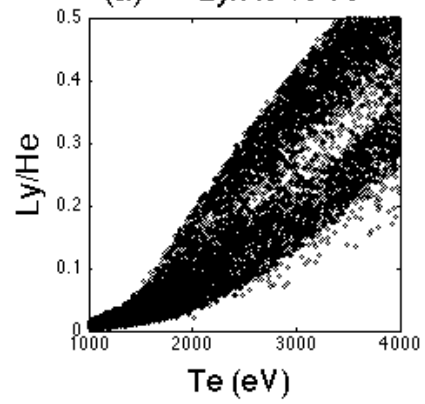

(e) Rll vs Te
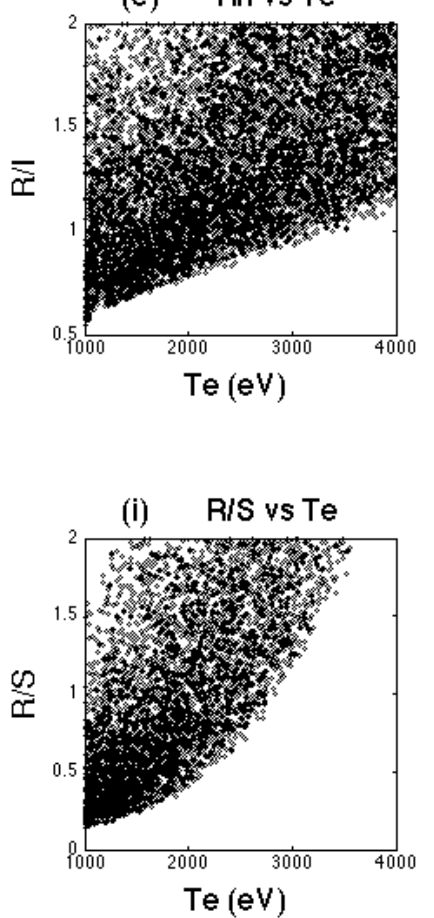

(b) Ly/He vs R/S

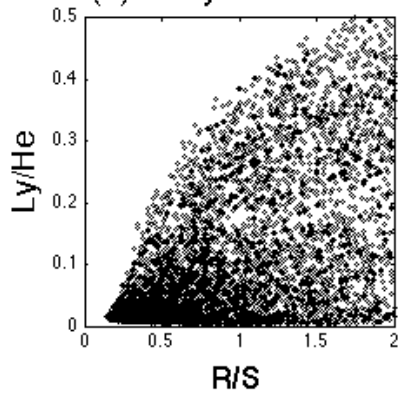

(f) RII vs Ti
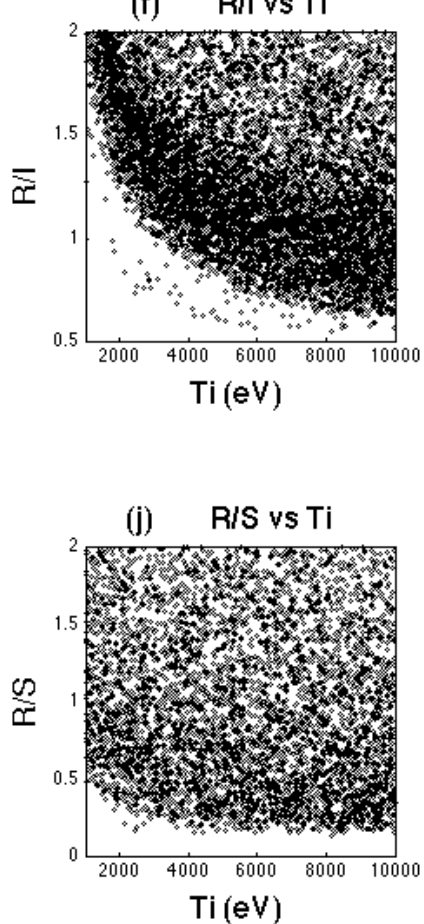

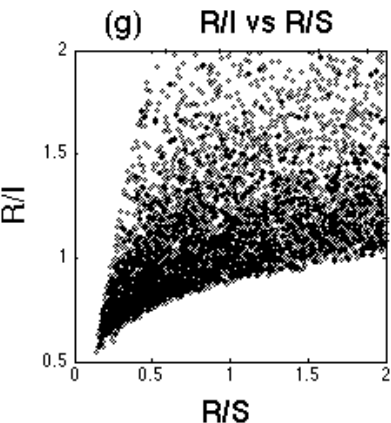

(k) R/S vs $\mathrm{Ne}$

(c) Ly/He vs R/l

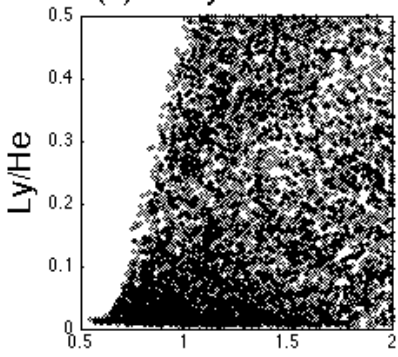

RII

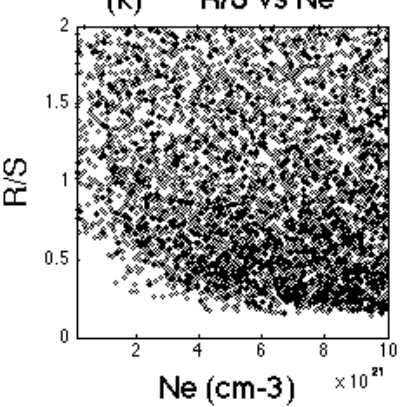

(d) LylHe vs e

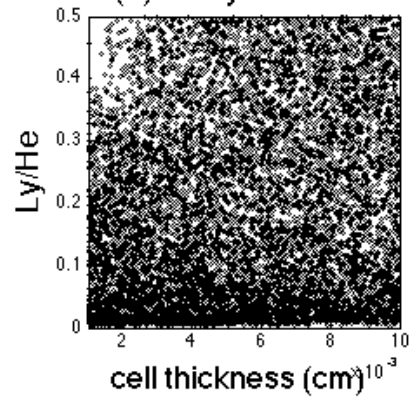

(h) RIl vse

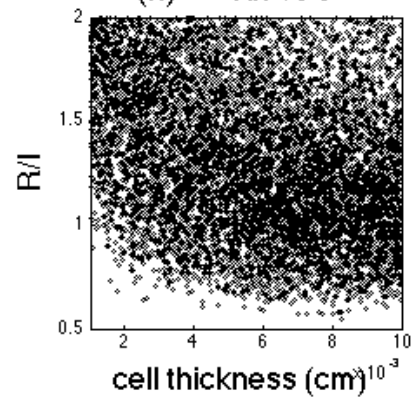

(I) RIS vse

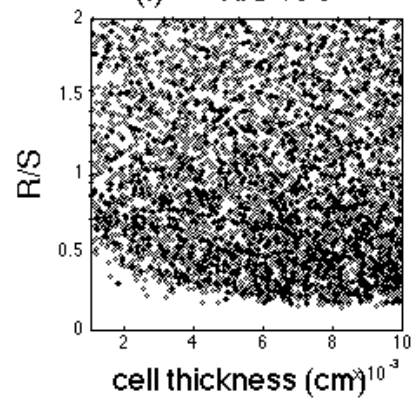

FIG. 17: Scatter plots of parameters dependence versus $T_{e}, T_{i}, N_{e}$ and cell thickness e: (a)-(d) for Ly- $\alpha / \mathrm{He}-\alpha,(\mathrm{e})-(\mathrm{h})$ for $R / I$ and (i)-(l) for $R / S$.(fichier matlab rand_rap.m)

agreement with those given by the FCI2 simulation.
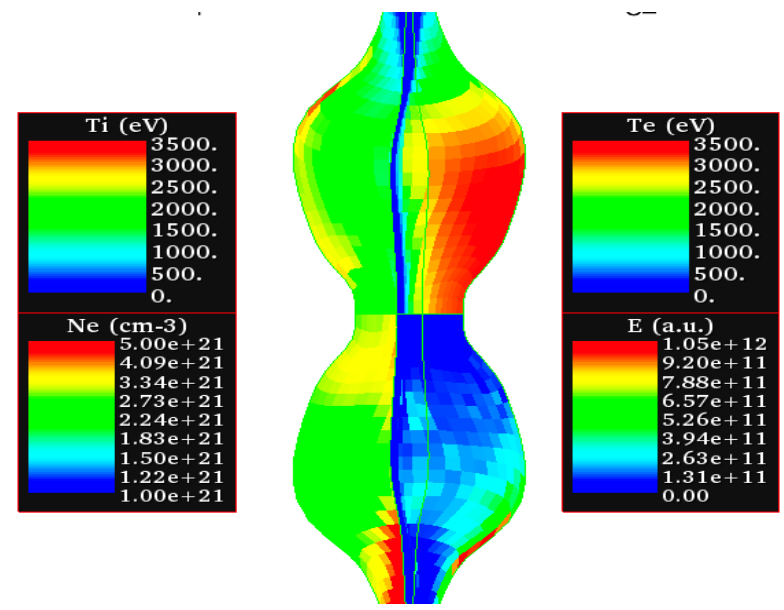

FIG. 18: Colormaps of $T_{i}$ (upper left), $T_{e}$ (upper right), $N_{e}$ (lower left) and emissivity (lower right), given by the simulation. 


\section{DISCUSSION}

It is interesting to compare spectra from targets with different aerogel densities to understand the origin of ionic temperature generated in the titanium foil. For this purpose it would have been appropriate to compare identical targets, one without aerogel and the other with aerogel at density 2 or $5 \mathrm{mg} / \mathrm{cm}^{3}$. Unfortunaltely, we did not get useful data for any such pair of shots. We can, however, compare shots \#58411 (SN5-3 $5 \mathrm{mg} / \mathrm{cc}$ aerogel-target type) and \#58412 (SN0-20 empty-target type). The spectra are shown on Figure 20 at time 1.1 ns of maximum emission. The $R / I$ ratios shown in Table IV decrease with aerogel density 1.468-1.313-1.289. The uncertainty on these values is large and depends on the spectrum quality that is worse for these shots since the spectrometer is looking at the rear-side of the foil which is thicker $(20 \mu \mathrm{m})$ for half of the shots. It is unlikely for these configuration of diagnostic that the dominant contribution to the measured spectrum also comes from a thin outer layer of the titanium plasma.

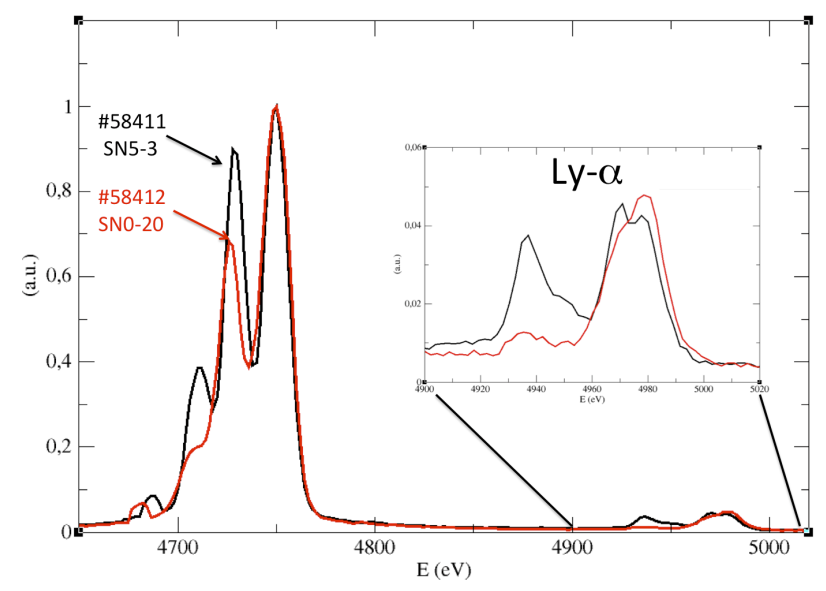

FIG. 20: Effect of the presence of aerogel on ionic temperature and therefore on the MSPEC spectrum.

\section{CONCLUSION}

We have been able to find thermodynamic conditions responsible for experimental spectral measured by the high resolution spectrometer MSPEC from novel hybrid target. It seems that we found favorable conditions where the measured spectra originate from a single emissive zone, consistent with $2 \mathrm{D}$ radiation-hydrodynamics simulations. The striking feature is that thermodynamic conditions have been found independently of any hypothesis on electron density or temperature. We only consider ratios of Ly- $\alpha$ to He- $\alpha$ integrals in addition to ratios of line amplitudes (Resonance, Intercombination and Satellite). A minimization procedure gave the best fit for all these ratios and delivers spectra very similar to the measured ones, provided that we introduced an ion temperature (greater than electronic temperature). The temperature has been found in most cases in reasonable agreement with the one calculated independently by 2D-radiationhydrodynamic simulations. A strong disagreement between the fitted values of $N_{e}$ and $T_{e}$ is an indication of the breakdown of our initial hypothesis, i.e. the localization of the emissive zone in a small definite outer region of the Titanium foil. In particular, for the June shots, when the MSPEC spectrometer is looking from behind the target, the multi-keV x-rays from the emissive zone cross a variable amount of cold or warm Titanium material. In that case we should take all the radiation path with varying conditions through the target into account in an integrated calculation with the TRANSPEC code. That is presumably why the ion temperature necessary to get the right spectra is higher than those given by 2D-simulations. We did not consider in this paper the possibility of cumulate spectra from multiple emissive zones because it did not seem to be necessary but the same optimization method could be used on good quality spectra. Initially these experiments were dedicated to the measurement $\mathrm{x}$-ray versus power with broadband diagnostics. Conditions have been found in which K-shell spectroscopy can bring a large amount of information and this is a very encouraging point. In a near future we will use a higher resolution spectrometer like XCCS-2 from CEA and we will design optimal configuration targets to infer more precise thermodynamic conditions from measured spectra. For example, a Thomson scattering measurement can give valuable information on $Z^{*} T_{e} / T_{i}$, independently and complementarily to other spectroscopic devices. The goal is still to validate the thermodynamics values calculated by ionizationtemperature-based simplified on-line NLTE models. Last point, the TRANSPEC code takes into account autoionization states and dielectronic capture processes. The ionization values calculated with and without these processes exhibit differences, in the thermodynamic conditions we have considered. These processes must be taken into account to reproduce all the K-shell structure in the range $4.65-6.5 \mathrm{keV}[27]$. This is important information by itself because we sometime invoked the role of two electron-processes as a part for a possible explanation of discrepancies between calculation and measurement in multi-keV x-ray sources especially when low Z material (from Titanium $(\mathrm{Z}=22)$ to Germanium $(\mathrm{Z}=32)$ ) are involved.

\section{ACKNOWLEDGMENTS}

We thank the OMEGA crew for their support. The plastic cylinders of targets have been built by CEAValduc, filled with low-density aerogel by J.H. Satcher and assembled by R.J. Wallace at LLNL whose work has been performed under the auspices of the US. Departement of Energy by Lawrence Livermore National Laboratory under contract No. DE-AC52-07NA27344. 

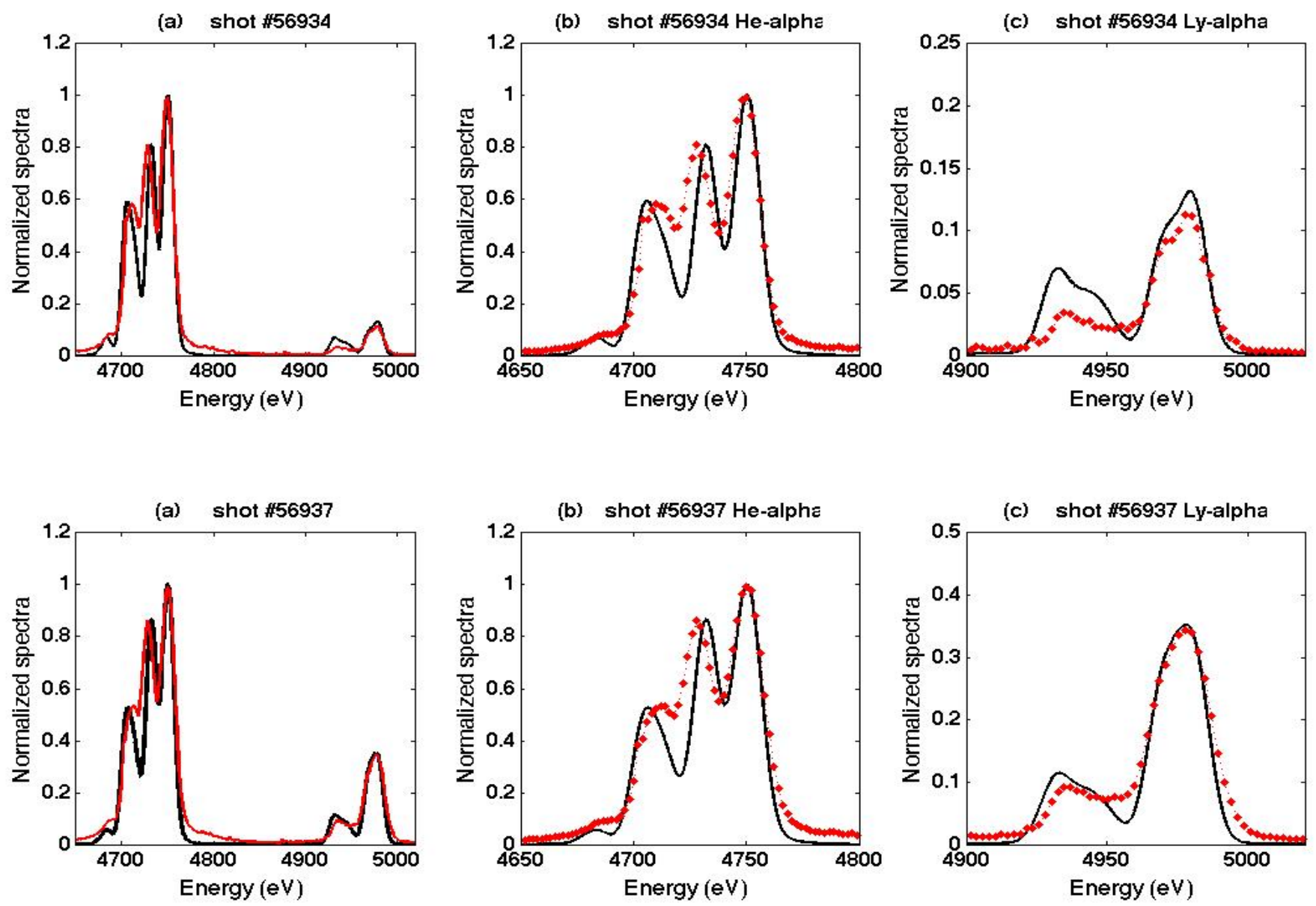

FIG. 19: Ly- $\alpha$ and He- $\alpha$ structures calculated by TRANSPEC superimposed with MSPEC spectra : shot \#56934 (upper curves) and shot \#56937 (lower curves) at $0.7 \mathrm{~ns}$.

[1] K. Fournier, J. Satcher, M. May, J. Poco, C. Sorce, J. Colvin, S. Hansen, S. MacLaren, S. Moon, J. Davis, et al., Physics of Plasmas 16, 052703 (2009).

[2] K. B. Fournier, M. J. May, J. D. Colvin, J. O. Kane, M. Schneider, E. Dewald, C. A. Thomas, S. Compton, R. E. Marrs, J. Moody, et al., Physics of Plasmas 17, 082701 (2010).

[3] L. Jacquet, M. Primout, B. Villette, F. Girard, and G. Oudot, High Energy Density Physics 9, 601 (2013).

[4] L. Jacquet, F. Girard, M. Primout, B. Villette, and P. Stemmler, Physics of Plasmas 19 (2012).

[5] F. Girard, M. Primout, B. Villette, P. Stemmler, L. Jacquet, D. Babonneau, and K. B. Fournier, Physics of Plasmas 16, 052704 (2009).

[6] D. Babonneau, M. Primout, F. Girard, J. P. Jadaud, M. Naudy, B. Villette, S. Depierreux, C. Blancard, G. Faussurier, K. B. Fournier, et al., Physics of Plasmas 15, 092702 (2008).
[7] M. Primout, JXSRT 5, 23 (2005).

[8] J. D. Colvin, K. B. Fournier, M. J. May, and H. A. Scott, Physics of Plasmas 17, 073111 (2010).

[9] J. D. Colvin, K. B. Fournier, J. Kane, S. Langer, M. J. May, and H. A. Scott, High Energy Density Physics 7, 263 (2011).

[10] J. L. Bourgade, B. Villette, J. L. Bocher, J. Y. Boutin, S. Chiche, N. Dague, D. Gontier, J. P. Jadaud, B. Savale, R. Wrobel, et al., Review of Scientific Instruments 72, 1173 (2001).

[11] J. P. Apruzese, K. G. Whitney, J. Davis, and P. C. Kepple, Journal of Quantitative Spectroscopy and Radiative Transfer 57, 41 (1997).

[12] C. Deeney, C. A. Coverdale, M. R. Douglas, T. J. Nash, R. B. Spielman, K. W. Struve, K. G. Whitney, J. W. Thornhill, J. P. Apruzese, R. W. Clark, et al., Physics of Plasmas 6, 2081 (1999).

[13] F. Girard, M. Primout, B. Villette, D. Brebion, 
H. Nishimura, and K. B. Fournier, High Energy Density Physics 7, 285 (2011).

[14] M. Primout, D. Babonneau, L. Jacquet, B. Villette, F. Girard, D. Brebion, P. Stemmler, K. Fournier, R. Marrs, M. May, et al., High Energy Density Physics 9, 750 (2013).

[15] M. May, R. Heeter, and J. Emig, Rev. Sci. Instrum 75, 3740 (2004).

[16] R. Heeter, J. Emig, K. Fournier, S. Hansen, M. May, and B. Young, Rev. Sci. Instrum 75, 3762 (2004).

[17] R. Marrs, G. Brown, J. Emig, and R. Heeter, Rev. Sci. Instrum 85, 11D626 (2014).

[18] L. Koppel and J. J. D. Eckels, UCRL 79781 (1977).

[19] O. Peyrusse, Phys. Of Fluids 56, 2007 (1992).

[20] O. Peyrusse, JQSRT 51, 281 (1994).

[21] O. Peyrusse, J. Phys. B:At. Mol. Opt. Phys. 33, 4303
(2000).

[22] D. Gilles and O. Peyrusse, JQSRT 53, 647 (1995).

[23] O. Peyrusse and A. Angelie, Ann. de Phys. 11, 157 (1986).

[24] M. Busquet, Physics of Fluids B-Plasma Physics 85, 4191 (1993).

[25] G. P. Schurtz, P. D. Nicolai, and M. Busquet, Physics of Plasmas 7, 4238 (2000).

[26] E. Buresi, J. Coutant, R. Dautray, M. Decroisette, B. Duborgel, P. Guillaneux, J. Launspach, P. Nelson, C. Patou, J. Reisse, et al., Laser Part. Beams 4, 531 (1986).

[27] M. Primout, D. Babonneau, B. Canaud, C. Blancard, and G. Faussurier, Journal of Quantitative Spectroscopy Radiative Transfer 71, 331 (2001). 